\title{
玉龙雪山地区地表三维温度场时空变化分析
}

\author{
谢俊峰 ${ }^{1,2,3}$, 莫 凡 ${ }^{1, *}$, 奚绍礼 ${ }^{4}$, 唐洪㻇 ${ }^{1}$, 褚 存 $^{2}$ \\ 1 自然资源部国土卫星遥感应用中心, 北京 100048 \\ 2 辽宁技术工程大学测绘与地理科学学院, 阜新 123000 \\ 3 重庆交通大学土木工程学院 重庆 400074 \\ 4 辽宁科技大学土木工程学院, 鞍山 114000
}

摘要:玉龙雪山属于海洋型冰川,对全球气候变化敏感,具有重要的生态研究价值。为了获取玉龙雪山地区的地表温度变化情 况,提出了一种基于地表三维温度场的定量分析方法。首先,采用高分辨率立体测绘卫星影像构建数字表面模型,作为三维底 图参考, 并利用多时序热红外卫星影像数据反演地表温度场模型, 提供地表温度变化依据; 然后,在统一参考坐标系下将数字表 面模型和多时序地表温度场模型套合,准确地分析地表温度时空变化情况。试验利用 1987 年至 2018 年间同一季节的 Landsat 卫星遥感影像反演地表温度,结合资源三号卫星立体影像构建的数字表面模型,并采用四阶温度区间分析多时序范围内玉龙雪 山地区的地表温度变化情况。试验结果表明玉龙雪山低温区和次低温区面积逐渐减小,减小速度分别为 $2.096 \mathrm{~km}^{2} / \mathrm{a}$ 和 2.662 $\mathrm{km}^{2} / \mathrm{a}$, 中温区和暖温区面积逐渐增大,增大速度分别为 $2.902 \mathrm{~km}^{2} / \mathrm{a}$ 和 $1.703 \mathrm{~km}^{2} / \mathrm{a}$,玉龙雪山地区的地表温度呈现整体上升的 趋势, 为全球生态环境变化提供参考。

关键词:玉龙雪山;数字表面模型;地表温度反演;三维温度场;时空变化

\section{Spatial and temporal variation of 3D temperature field in Yulong Snow Mountain area}

\author{
XIE Junfeng ${ }^{1,2,3}$, MO Fan ${ }^{1, *}$, XI Shaoli ${ }^{4}$, TANG Hongzhao ${ }^{1}$, CHU Cun ${ }^{2}$ \\ 1 Land Satellite Remote Sensing Application Center, Ministry of Natural Resources, Beïing 100048, China \\ 2 School of Surveying and Geographical Science, Liaoning Technical University, Fuxin 123000, China \\ 3 College of Civil Engineering, Chongqing Jiaotong University, Chongqing 400074, China \\ 4 College of Civil Engineering, Liaoning University of Science and Technology, Anshan 114000, China
}

\begin{abstract}
The Yulong Snow Mountain is characterized by marine glaciers, which is sensitive to earth's climate change and has important ecological research value. To study the variation of surface temperature in the Yulong Snow Mountain area, a quantitative analysis method based on a three-dimensional (3D) surface temperature field is proposed in this paper. The digital surface model is constructed using high-resolution stereo mapping satellite images to provide accurate 3D terrain reference. Multi-temporal thermal infrared satellite image data are used to invert the surface temperature field model to provide a basis for the change of surface temperature. The digital surface model is combined with the multi-temporal surface temperature field model to analyze the spatio-temporal variation of surface temperature in detail. Based on Landsat satellite images acquired by same season from 1987 to 2018, a digital surface model is constructed by inversion of surface temperature and 3D images of the ZY3 satellite. The fourth order temperature interval was used to analyze the surface temperature variation in the Yulong Snow Mountain. The low temperature area and the sub-low temperature area are
\end{abstract}

基金项目:国家国防科技工业局预先研究项目(D040105, D040106); 科技部重点研发计划(2018YFB0504800); 科技部重点研发计划国际合作 项目 (2020YFE0200800)

收稿日期: 2020-02-23; 网络出版日期:2021-01-27

* 通讯作者 Corresponding author.E-mail: surveymofan@163.com 
shrinking with a speed of $2.096 \mathrm{~km}^{2} / \mathrm{a}$ and $2.662 \mathrm{~km}^{2} / \mathrm{a}$, respectively. The medium temperature area and the warm temperature area are expanding with a speed of $2.902 \mathrm{~km}^{2} / \mathrm{a}$ and $1.703 \mathrm{~km}^{2} / \mathrm{a}$ within the experimental area in recent years. The multi-temporal surface temperature changes in the Yulong Snow Mountain area are analyzed. The results show that the climate is warming around the Yulong Snow Mountain area, and could provide reference for global ecological environment change.

Key Words: Yulong Snow Mountain; digital surface model; surface temperature inversion; three-dimension temperature field; spatial and temporal variation

玉龙雪山位于我国的云南省丽江市, 呈南北走向, 海拔最高为 5500 余米, 其温度气候呈现垂直差异, 山顶 终年积雪, 区域温差较大。玉龙雪山自然保护区留有我国珍贵的古冰川遗迹,育有完整自然生态系统,具有极 其重要的科研及旅游价值 ${ }^{[1]}$ 。由于其特殊的地理位置, 玉龙雪山对全球气候变化趋势敏感性较强, 因此玉龙 雪山的生态环境变化可以很好地反映全球气候变化 ${ }^{[2]}$ 。

陆地表面温度是生态环境分析等研究应用的关键指标,在全球环境变化和生态资源监测等领域具有重要 的研究价值 ${ }^{[3]}$ 。随着卫星遥感技术的不断发展, 卫星平台的承载力逐渐增大、稳定度不断提高, 为大区域陆 地表面温度定量化地反演提供了一种有效的技术手段。目前, 利用卫星热红外遥感影像反演陆地表面温度在 地矿资源的探测、城市热岛效应分析、植被病虫害状态监测等领域都取得了重要的研究成果。黄秀华等利用 不同时相下热红外遥感数据提取热异常标志, 指出了找油的最佳时机 ${ }^{[4]}$ 。张小飞通过提取相关的下垫面类 型、地表温度和植被覆盖等信息, 证实城市区域植被覆盖状况可直接影响城市地表温度 ${ }^{[5]}$ 。毛克彪提出利用 MODIS 数据基于辐射传输方程来进行地表温度反演 ${ }^{[6]}$ 。周纪构建立了城市热岛容量计算模型,综合城市热 岛强度、足迹面积等多方面信息, 在最大程度上定量地反映和刻画了热岛效应发生的显著程度, 能够客观描 述城市热岛对局地气候、人居环境质量的影响 ${ }^{[7]}$ 。徐永明利用 MOIDS 数据提出了改进的温度-植被指数, 在 农田区域及农作物生长期内具有很好的适用性和精度, 为有效获取大范围农田气温提供了新的思路 ${ }^{[8]}$ 。 Brabyn Lars 从 Landsat 7 ETM +影像提取地表温度,指出利用 Landsat 7 ETM +得出的地表温度可广泛地应用 于生态和气象研究 ${ }^{[9]}$ 。卢显等基于中国 HJ-1B 卫星热红外数据验证了 2010 年 4 月 14 日青海玉树 M_S7.1 地 震 LST 临震地表温度异常的可靠性 ${ }^{[10]}$ 。冯海英等人利用 MODIS 反演的地表温度和土地覆盖产品提出一种 评估森林调节温度生态服务价值的新方法, 为正确认识和定量评估森林生态服务价值及进行生态系统管理提 供参考 ${ }^{[11]}$ 。吴文波等利用 Landsat 8 OLI/TIRS 遥感影像反演地表温度,结果表明地表热环境分布与断裂带等 自然因素存在一定相关性 ${ }^{[12]}$ 。目前, 基于两线阵、多线阵传感器获取的立体像对重建高精度数字表面模型的 研究也取得了很大的进步 ${ }^{[13]}$ 。林行刚基于 DSM 讨论了精确地形对飞行器跟踪制导技术的重要工程应用前 景 ${ }^{[14]}$ 。王明华采用 DSM 完成了工程岩体三维地质建模与可视化 ${ }^{[15]}$ 。穆超等基于高分辨率遥感影像, 实现 了 DSM 建筑物点的提取, 对建筑物的精确三维重建提供了有效帮助。Ola Friman 等采用数字表面模型来估 计人射光的不同分量, 进而预测不同光照条件下的被测光谱 ${ }^{[16]}$ 。Mathieu Brédifd 等提出一种从数字表面模型 提取建筑物占地面积的全自动框架, 给出了利用 DSM 构建 $3 \mathrm{D}$ 城市模型的全自动过程 ${ }^{[17]}$ 。Lasse Sander 等利 用 SRTM 数字地面模型获取到宽滩脊系统的海拔趋势 ${ }^{[18]}$ 。凌成星等提出了一种基于 GeoEye- 1 立体像对提 取平均树高的方法, 可快速获得研究区大范围森林平均树高 ${ }^{[19]}$ 。解金卫等人提出一种高精度 DSM 估计方 法, 能有效提高植被区 DSM 反演精度 ${ }^{[20]}$ 。

热红外遥感能够获取地表温度信息, 可见光遥感具有出色的地面分辨率, 在对地进行精确定位及立体成 像方面具有无可比拟的优势。现阶段围绕数字表面模型以及热红外遥感地表温度反演两个领域的应用研究 都有了很多重要成果,但是通过两者的融合应用开展多维遥感信息探测还处于初始阶段,尚有大量空白空间 需要国内外学者进行探索。将星载热红外数据与可见光数据融合使用, 突破单一数据的桎梏, 可进一步拓宽 并提升遥感应用的边界与高度, 构建地表三维温度场。相比较单一的热红外遥感应用, 更真实地反应研究区 
的实际地表情况,结合温度信息可以实现立体式热红外探测,通过利用多维信息主被动复合观测,显著提升目 标多维度信息综合监测能力。

本文从星载热红外与可见光立体遥感数据融合应用出发, 以研究玉龙雪山的温度变化为目的,基于 Landsat 系列卫星的热红外数据反演二维地表温度,基于资源三号卫星的三线阵可见光影像构建数字表面模 型,通过两者嵌套完成地表三维温度场构建,定量分析研究区域的地表三维温度场时空变化。

\section{1 数据与方法}

\section{1 数据}

研究数据选取区域为玉龙雪山区域,大致位于我国 丽江市内 $100^{\circ} 4^{\prime} 2^{\prime \prime} \mathrm{E}$ 至 $100^{\circ} 16^{\prime} 30^{\prime \prime} \mathrm{E} 、 27^{\circ} 3^{\prime} 2^{\prime \prime} \mathrm{N}$ 至 $27^{\circ} 18^{\prime}$ $57^{\prime \prime} \mathrm{N}$ 处,地理位置如图 1 所示。研究数据选用陆地卫 星 (Landsat) 系列的热红外影像以及立体测绘卫星资源 三号 $(\mathrm{ZY} 3)$ 的三线阵影像为基础研究数据。Landsat TM 以及 Landsat 8 TIRS 提供了从 1984 年至今的热红 外数据,本文选取了从 1987 年至 2018 年的时间跨度 中, 以两到三年为时间间隔的 13 个年份中每年的 12 月 热红外影像数据进行处理, 并且通过对比选择该月份中 质量较高的日期的影像数据 (由于数据质量原因, 在 2004 年至 2008 年的数据为四年的时间间隔)。数据在

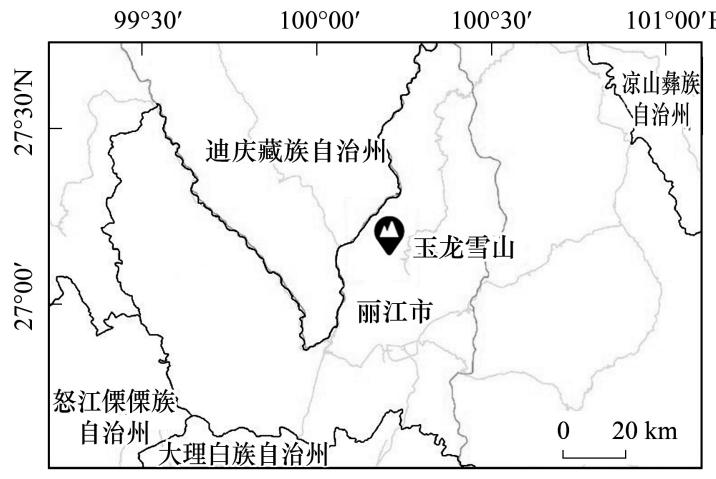

图 1 玉龙雪山地理位置示意图

Fig.1 Geographical location of Yulong Snow Mountain 美国地质勘探局网站( USGS) 下载得到。资源三号卫星的三线阵影像数据包括前视、后视及全色影像, 轨道行 列号 Path 为 33 , Row 为 157 , 数据通过自然资源部国土卫星遥感应用中心获取得到。由于较早时期的热红外 影像数据无相对应的资源三号数据, 以相近时相下的 Landsat ETM+ 全色影像模拟为高分辨率可见光影像对 其进行影像配准。以上数据均以 Geotiff 格式储存,采用了 UTM-WGS84 坐标系统进行地图投影。

1.2 方法

\subsection{1 普适性单通道算法反演地表温度}

普适性单通道算法 (generalized single-channel method，GSC) 顾及了地表比辐射率和大气辐射参数, 是一 种可靠性较高的温度反演算法。其可适用于 Landsat 4 TM 热红外数据、Landsat 7 ETM+热红外数据和 Landsat 8 TIRS 数据,算法公式如下 ${ }^{[21-22]}$ :

$$
\begin{gathered}
T_{S}=\gamma\left[\frac{1}{\varepsilon_{i}}\left(\psi_{1} L_{i}+\psi_{2}\right)+\psi_{3}\right]+\delta \\
\gamma \approx \frac{T_{i}^{2}}{b_{\gamma} L_{i}} \\
\delta \approx T_{i}-\frac{T_{i}^{2}}{b_{\gamma}}
\end{gathered}
$$

式中, $T_{S}$ 为地表温度, 单位为 $\mathrm{K}_{\circ} T_{i}$ 为波段通道的亮度温度, 单位为 $\mathrm{K}_{\circ} L_{i}$ 为大气顶层卫星传感器所接收到的 辐射亮度, 单位为 $\mathrm{W} \mathrm{m}^{-2} \mathrm{sr}^{-1} \mu \mathrm{m}^{-1} ; b_{\gamma}=c_{2} / \lambda, c_{2}$ 为普朗克函数中的常量, $c_{2}=1.43877 \times 10^{4} \mu \mathrm{m} \cdot \mathrm{K}, \lambda$ 是有效波 长。对于 Landsat $4 \mathrm{TM}$ 第 6 波段, $b_{\gamma}$ 为 $1290 \mathrm{~K}$ 。对于 Landsat $5 \mathrm{TM}$ 第 6 波段, $b_{\gamma}$ 为 $1256 \mathrm{~K}$ 。对于 Landsat 7 $\mathrm{ETM}+$ 第 6 波段, $b_{\gamma}$ 为 $1277 \mathrm{~K}$ 。对于 Landsat8 TIRS 第 10 波段, $b_{\gamma}$ 为 $1324 \mathrm{~K}_{\circ} \Psi_{1}$ 、 $\Psi_{2}$ 及 $\Psi_{3}$ 是关于大气水汽含 量 $\omega$ 的大气参数, 由下式及表中的计算系数利用大气水汽含量计算得到:

$$
\left[\begin{array}{l}
\psi_{1} \\
\psi_{2} \\
\psi_{3}
\end{array}\right]=\left[\begin{array}{lll}
c_{11} & c_{12} & c_{13} \\
c_{21} & c_{22} & c_{23} \\
c_{31} & c_{32} & c_{33}
\end{array}\right]\left[\begin{array}{c}
w^{2} \\
w \\
1
\end{array}\right]
$$




\subsection{2 铅垂线轨迹法制作数字表面模型}

铅垂线轨迹法 (VLL-Vertical Line Locus) 是制 作数字表面模型过程中常用的技术策略之一。其基本 思想是: 假设在物方有一条铅垂线轨迹,则它在画幅式 像片上的投影也是一条直线,铅垂线与地面交点 $\mathrm{A}$ 在 像片上的构像必定位于相应的投影辐射线上,原理示意 图如下 ${ }^{[13,23]}$ 。

利用铅垂线轨迹法搜索其相应的像点 $a_{1}$ 和 $a_{2}$, 确 定 $\mathrm{A}$ 点高程的基本计算步骤如下: 给定地面点的平面 坐标 $\left(X_{0}, Y_{0}\right)$ 与可能的最低高程 $Z_{\text {min }}$, 高程搜索步距 $d Z$ 可以按照所要求的高程精度决定。由地面点的平面 坐标 $\left(X_{0}, Y_{0}\right)$ 与可能的高程: $Z_{i}=Z_{\text {min }}+i \cdot d Z,(i=0$, $1, \cdots n)$, 计算地面点分别在左右像片上的像点坐标 $\left(x_{1}, y_{1}\right),\left(x_{2}, y_{2}\right)$ 。分别以 $\left(x_{1}, y_{1}\right) 、\left(x_{2}, y_{2}\right)$ 为中心在 各自影像上选取匹配窗口, 计算其匹配测度, 如计算相 关系数 $\rho_{i}$ (也可以利用其他测度)。令 $i=i+1$, 重复上

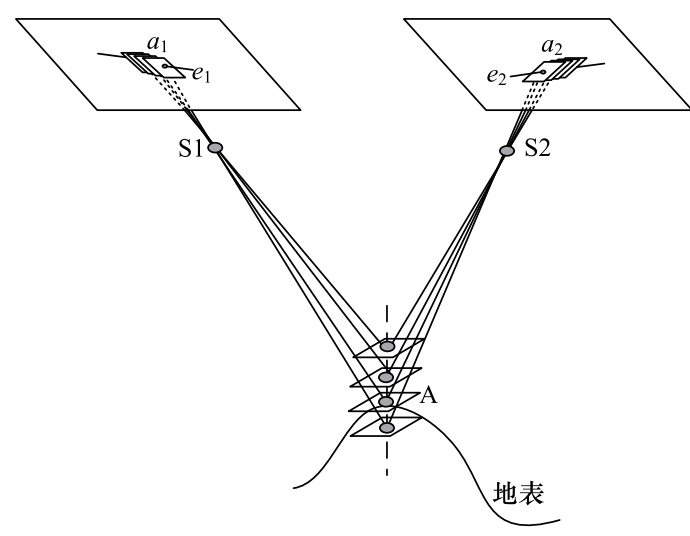

图 2 铅垂线轨迹法匹配制作数字表面模型

Fig.2 DSM produced based on VLL $a_{1}$ : 左影像上像点, $a_{2}$ : 右影像上对应同名像点; $e_{1}$ 和 $e_{2}$ : 像点 $a_{1}$ 和 $a_{2}$ 构成的像方轨迹线; $s_{1}$ 和 $s_{2}$ : 摄影的焦点; $A$ 为地面点 述步骤, 得到一组匹配测度 $\left\{\rho_{0}, \rho_{1}, \rho_{2}, \cdots, \rho_{k}\right\}$, 选取其中的最大值 $\rho_{k}$, 其对应的高程为 $Z_{k}=Z_{\text {min }}+k \cdot d Z$, 则 认为地面点 $\mathrm{P}$ 的高程值为 $Z_{k}$ 。还可以利用 $\rho_{k}$ 及其相邻的几个相关系数拟合一条抛物线, 以其极值对应的高 程作为地面点的高程, 以进一步提高精度,或以更小的高程步距 $d Z$, 在一小范围内重复以上过程。

\subsection{3 三维温度场构建}

地表三维温度场作为星载热红外数据与可见光数据融合产品,具有几何高精度、信息多维度的优势。在 构建地表三维温度场时, 根据应用需求,在数据选择方面需要考虑热红外数据的时间分辨率和温度分辨率,例 如 Landsat 系列卫星在理想状态下获取从 1984 年至今的 16 天时间分辨率平均误差值在 $1 \mathrm{~K}$ 左右的地表温度 数据, 可广泛应用于各种热红外遥感的研究应用。资源三号卫星三线阵影像具有高精度的几何定位的能力, 采用同轨立体像对生产数字表面模型, 为地表三维温度场提供精确可靠的地面三维坐标数据。

三维温度场的构建基本流程如下:首先对原始可见光影像数据进行预处理,消除影像的几何与辐射畸变 等。采用热红外数据反演地表温度,得到表征地表温度信息如图 3 所示的影像数据。使用可见光数据的前 视、后视及正视影像制作如图 3 的数字表面模型。最后将热红外温度影像与数字表面模型在统一地理基准的
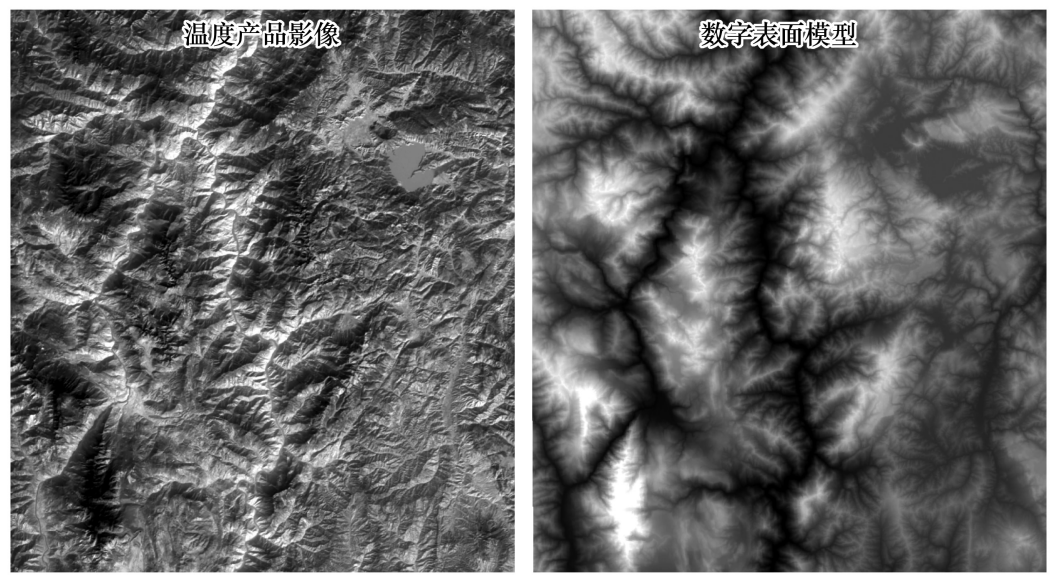

图 3 温度反演与立体影像产品

Fig.3 Image data after temperature inversion and stereo imaging 
情况下进行套合, 完成热红外与可见光遥感数据地融 合,得到既包含了温度信息又具有高精度三维坐标如图 4 所示的地表温度场数据,技术流程如图 5 所示。

\section{3 结果与分析}

利用普适性单通道算法进行温度反演,在获得研究 区域的一个时间序列的地表温度反演结果后,为了更好 的研究分析该区域地表三维温度场的时间演变规律及 空间分布特征,需要对地表温度产品进行温度等级的划

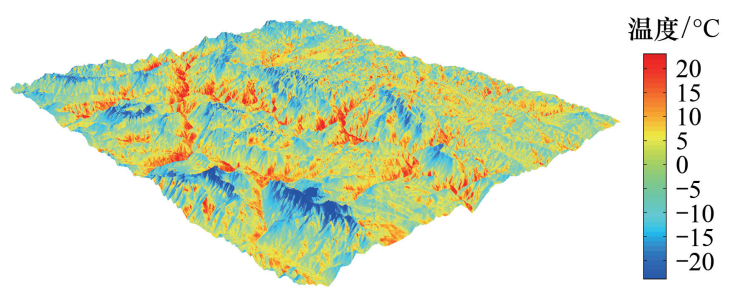

图 4 地表三维温度场

Fig.4 Surface three-dimensional temperature field 分。通过对不同温度区间进行分层赋色,可以直观的表现出该研究区域中不同温度区间的覆盖范围及相对分 布情况。本文通过密度分割并基于等间距分割法来划分地表温度等级,划分低温等级后的温度产品结果如图 6 所示:

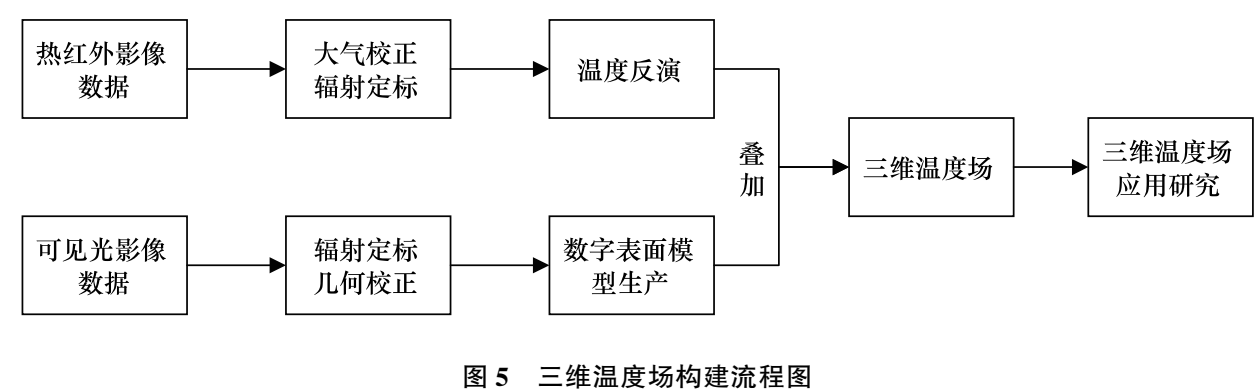

Fig.5 Three-dimensional temperature field building workflow

将以上反演得到的地表温度产品, 与 DSM 进行融合叠加处理, 获得所有温度产品对应的地表三维温度 场。如图 7 所示,不仅可以看出最终生成的地表三维温度场直观明了地展现出玉龙雪山区域在 1987 年到 2018 年时间序列中具体地表的温度情况, 同时,还可以获取到不同地形地物的具体温度信息及三维坐标位置 信息。

为了方便对玉龙雪山区域的地表三维温度场进行更直观的定量分析,根据影像获取时刻的地表温度,将 研究区域简单划分为四个温度区间: 低温区 $\left(T<-10^{\circ} \mathrm{C}\right)$ 、次低温区 $\left(-10^{\circ} \mathrm{C}<T<0^{\circ} \mathrm{C}\right)$ 、中温区 $\left(0^{\circ} \mathrm{C}<T<10^{\circ} \mathrm{C}\right)$ 以 及暖温区 $\left(T>10^{\circ} \mathrm{C}\right)$ 。由于地表三维温度场具有地面及高程的高精度坐标信息,可以计算得到不同于平面投 影面积的真实地表面积,通过温度属性进行分割可以计算得到不同温区的真实地表面积。对每个温区的地表 投影及实际面积进行统计分析对比 (表 1、表 2), 并以 1987 年至 2018 年中的 12 月为时间序列绘制不同温区 地表面积趋势折线图。

根据上述图表给出的结果,对玉龙雪山区域从 1987-2018 年的地表三维温度场的时空变化特征进行研 究分析:

(1) 从图 6 玉龙雪山区域历年温度平面分布图可以看出,由于研究区域中部的海拔较高,温度一致呈现 出由中间区域向外递减的趋势, 图 7 玉龙雪山区域历年地表三维温度场则更直观的呈现出了历年温度最低的 区域一致集中在了海拔最高的山峰顶部,而在海拔较低及人类活动区域温度逐渐升高。

(2) 由图 8 和表 3 可以看出, 针对玉龙雪山的时空变化分析,地表三维温度场相较于二维数据分析优势 明显,对不同温区真实地表面积变化情况更加敏感。其中次低温区位于高程变化显著区域,真实地表面积与 投影面积差异最为明显, 中温区次之。基于地表三维温度场,充分利用地形高程信息,可以表现出不同二维数 据的真实地表情况。玉龙雪山从河谷到山顶高程差距较大, 对于其垂直气候的分层差异, 表现出不同高程下 育有不同的气候带。由于地表三维温度场相比较平面温度场在统计地表面积方面会因高程差异显著更为精 


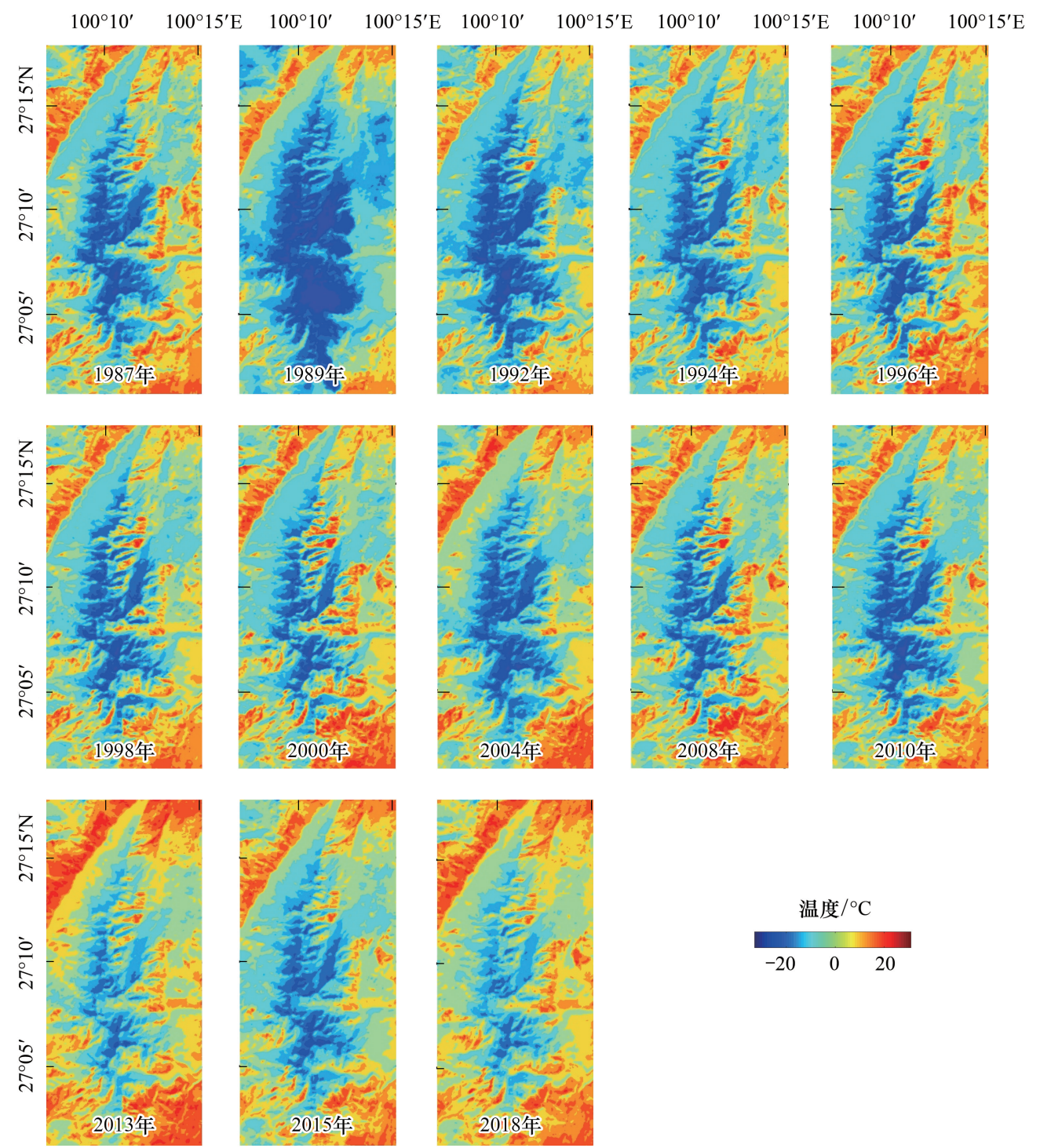

图 6 玉龙雪山区域历年温度平面分布图

Fig.6 Temperature distribution map of the Yulong Snow Mountain area over the years

确,因此建立地表三维温度场对于玉龙雪山等山地区域的生态研究分析意义重大。

(3) 在 1987-2018 年间,研究区不同温区的地表面积变化呈现出不稳定的变化现象,整体变化趋于温度 升高。由表 2 及图 8 可知,所选取的 13 年中, 1989 年玉龙雪山的整体温度分布最低,低温区 $\left(T<-10^{\circ} \mathrm{C}\right)$ 所占 面积大幅度提高, 之后呈现出逐年递减并在 1994 年后趋于稳定的状态。次低温区 $\left(-10^{\circ} \mathrm{C}<T<0^{\circ} \mathrm{C}\right)$ 从 1992 年 升至最高, 在 1989 年至 1994 年相对稳定,之后表现出逐年下降的趋势。中温区 $\left(0^{\circ} \mathrm{C}<T<10^{\circ} \mathrm{C}\right)$ 在 1989 年降 至最低后,总体表现出先是稳定增加的现象,在 2008 年后,温度分布出现不稳定的状态。相应的,在 1996 年, 玉龙雪山的暖温区 $\left(T>10^{\circ} \mathrm{C}\right)$ 地表面积呈现出明显上升, 并在之后的较长的之间序列中变化较小, 但是在 2000 年后, 不稳定性增强, 年间温度变化较大。从以上现象可以看出, 随着全球温度上升的趋势愈加显著, 玉 龙雪山及类似山地或者冰川的自然生态所受影响也愈加明显。同时, 根据相关研究表明, 喜马拉雅山冰川于 1990-2015 年也呈现出持续退缩状态 ${ }^{[24]}$, 并且其变化会随着高程有所不同。全球变暖造成的影响是整体性 的, 同时与高程也密切相关, 本文方法对雪山或冰川相关的生态环境的科学研究有着一定的可行性及优势。 

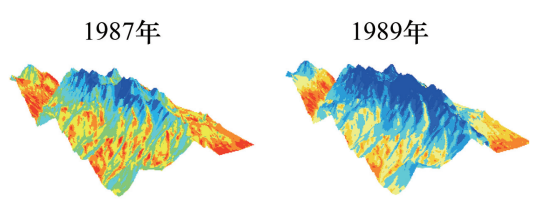

1992年

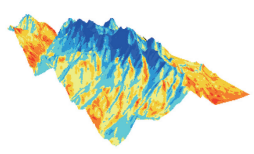

2004年

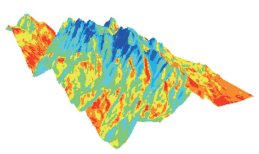

2013年

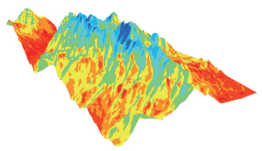

2000年

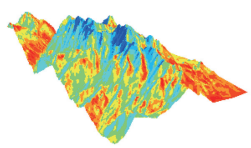

2015年

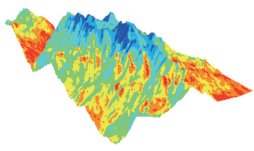

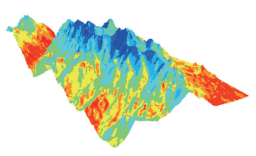

2018年

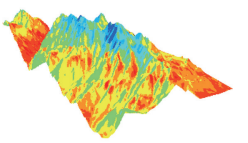

1994年

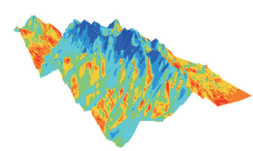

2008年
1996年

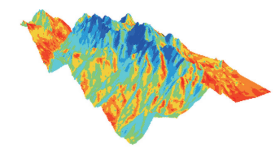

2010年

图 7 玉龙雪山区域历年地表三维温度场

Fig.7 Surface three-dimensional temperature field of the Yulong Snow Mountain area over the years

\section{表 1 研究区历年不同温区地表投影面积统计表}

Table 1 Statistical table of surface area in different temperature zones in the study area over the years

\begin{tabular}{|c|c|c|c|c|}
\hline \multirow{3}{*}{$\begin{array}{l}\text { 年份 } \\
\text { Year }\end{array}$} & \multicolumn{4}{|c|}{$\begin{array}{c}\text { 地表投影面积 } / \mathrm{m}^{2} \\
\text { Surface projected area }\end{array}$} \\
\hline & 低温区 & 次低温区 & 中温区 & 暖温区 \\
\hline & Low temperature area & Sub-low temperature area & Medium temperature area & Warm temperature area \\
\hline 1987 & 39662614.90 & 109303632.65 & 159855686.73 & 67870813.35 \\
\hline 1989 & 100350516.75 & 167894283.54 & 103698850.58 & 26357530.22 \\
\hline 1992 & 59986222.59 & 166272893.98 & 129727603.83 & 27822318.12 \\
\hline 1994 & 36772838.41 & 156952932.20 & 148880592.49 & 39377098.06 \\
\hline 1996 & 32347327.41 & 116767734.97 & 151196739.69 & 79356377.08 \\
\hline 1998 & 36104902.05 & 121533270.57 & 166544567.57 & 56728735.02 \\
\hline 2000 & 25584904.35 & 118287895.83 & 158910885.55 & 76465735.39 \\
\hline 2004 & 42798974.11 & 106557479.78 & 178855534.53 & 63030005.13 \\
\hline 2008 & 25203350.03 & 94355528.34 & 182044671.10 & 80184375.92 \\
\hline 2010 & 39429875.41 & 122682259.54 & 164257837.26 & 56664710.04 \\
\hline 2013 & 8431398.76 & 71556576.18 & 210857646.13 & 102686563.61 \\
\hline 2015 & 25610860.42 & 117031621.74 & 186285028.76 & 61136941.97 \\
\hline 2018 & 6038248.54 & 70203399.40 & 223005089.81 & 94995778.22 \\
\hline
\end{tabular}

\section{表 2 研究区历年不同温区地表实际面积统计表}

Table 2 Statistical table of surface area in different temperature zones in the study area over the years

\begin{tabular}{|c|c|c|c|c|}
\hline \multirow{3}{*}{$\begin{array}{l}\text { 年份 } \\
\text { Year }\end{array}$} & \multicolumn{4}{|c|}{$\begin{array}{l}\text { 地表实际面积 } / \mathrm{m}^{2} \\
\text { Actual surface area }\end{array}$} \\
\hline & 低温区 & 次低温区 & 中温区 & 暖温区 \\
\hline & Low temperature area & Sub-low temperature area & Medium temperature area & Warm temperature area \\
\hline 1987 & 52896338.98 & 147788068.03 & 190471082.10 & 81133720.98 \\
\hline 1989 & 133426648.73 & 209607304.21 & 126070284.26 & 30779350.16 \\
\hline 1992 & 79977864.14 & 214958033.35 & 153384006.34 & 33733518.01 \\
\hline 1994 & 49403149.25 & 205883234.46 & 176199607.86 & 48063533.86 \\
\hline 1996 & 43814895.08 & 158199116.71 & 179079397.20 & 95500285.68 \\
\hline 1998 & 49325713.55 & 162151764.34 & 197769789.54 & 68358638.94 \\
\hline 2000 & 34756609.54 & 158932294.55 & 188717199.36 & 92952879.26 \\
\hline 2004 & 56911242.63 & 137386290.47 & 222593681.08 & 73886064.30 \\
\hline 2008 & 34076350.09 & 129156315.29 & 217958993.70 & 97110029.61 \\
\hline 2010 & 52734908.29 & 163877200.62 & 195775209.50 & 67567869.08 \\
\hline 2013 & 11477557.69 & 96139363.52 & 263648243.73 & 122198348.58 \\
\hline 2015 & 34344081.36 & 156072114.10 & 226604381.22 & 72053917.57 \\
\hline 2018 & 11961364.96 & 95602920.82 & 236784500.70 & 113642608.88 \\
\hline
\end{tabular}


表 3 不同温区地表面积变化斜率

Table 3 Slope of surface area change in different temperature zones

\begin{tabular}{|c|c|c|c|c|}
\hline & $\begin{array}{c}\text { 低温区 } \\
\text { Low temperature } \\
\text { area }\end{array}$ & $\begin{array}{c}\text { 次低温区 } \\
\text { Sub-low temperature } \\
\text { area }\end{array}$ & $\begin{array}{c}\text { 中温区 } \\
\text { Medium temperature } \\
\text { area }\end{array}$ & $\begin{array}{c}\text { 暖温区 } \\
\text { Warm temperature } \\
\text { area }\end{array}$ \\
\hline $\begin{array}{l}\text { 实际面积变化趋势 } \\
\text { Actual area change trend }\end{array}$ & -2.096 & -2.662 & 2.902 & 1.703 \\
\hline $\begin{array}{l}\text { 投影面积变化趋势 } \\
\text { Projected area change trend }\end{array}$ & -1.610 & -2.164 & 2.596 & 1.443 \\
\hline
\end{tabular}
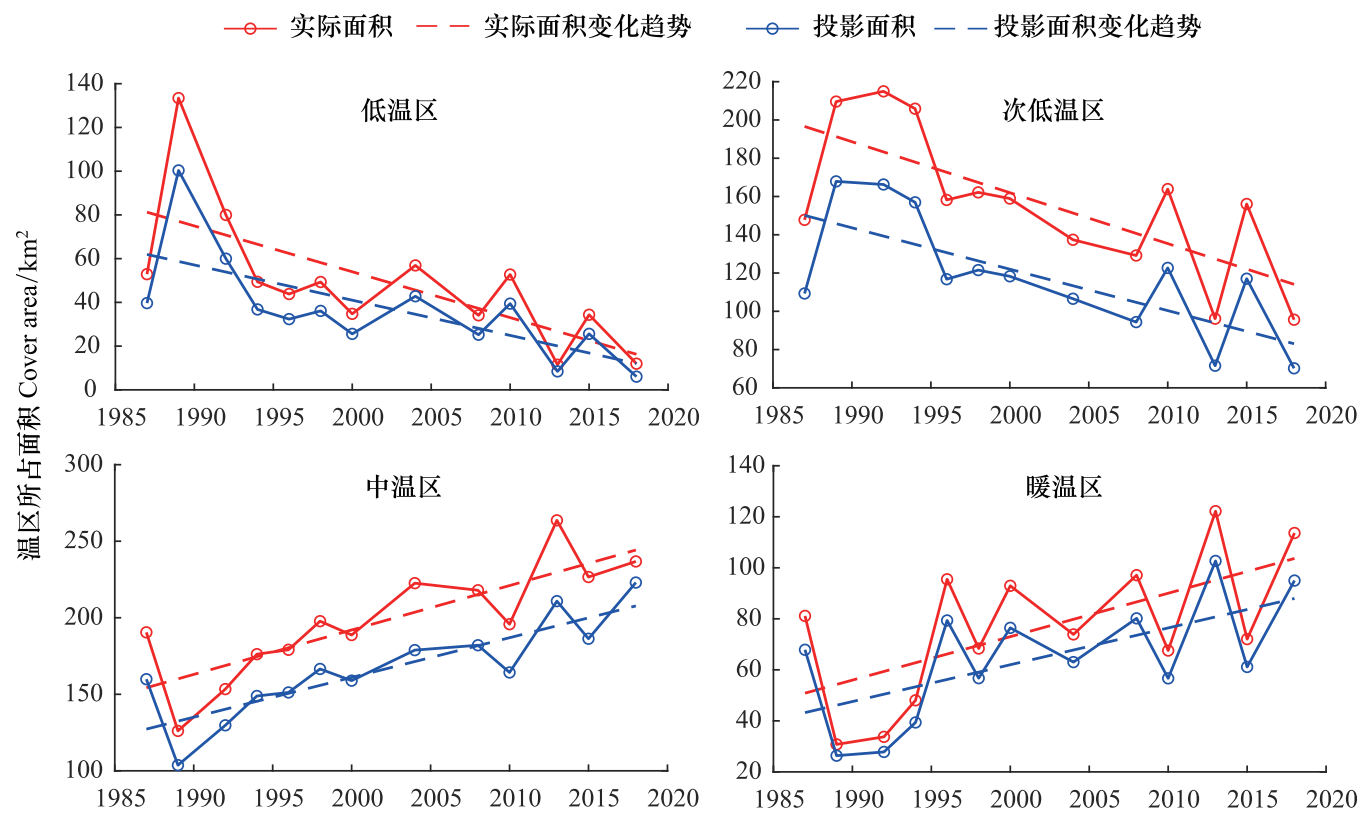

年份 Year

图 8 不同温区地表面积变化总体趋势图

Fig.8 Trend map of surface area change in different temperature zones

\section{4 结论}

本文利用陆地系列卫星和资源三号卫星的影像数据, 通过套合温度反演产品和数字表面模型, 构建了云 南省丽江市玉龙雪山区域的地表三维温度场,研究分析了该区域由 1987-2018 年的时空变化特征,并分析不 同温区的面积变化。根据本文地表三维温度场与二维数据比较结果, 针对高程起伏明显区域,地表三维温度 场优势明显, 能够更加有效地反映地表温度情况, 对于山地区域的研究具有重要意义。同时, 玉龙雪山区域研 究时间序列内的中温区和暖温区的地表面积有逐年增加的趋势,相较之下中温区的地表面积升幅稍大; 低温 区和次低温区的地表面积大体呈现减少的趋势; 可以发现玉龙雪山地区的整体温度变暖, 验证了全球温度变 暖的趋势。

\section{参考文献 (References) :}

[ 1 ] 杨绕琼, 范泽金金, 李宗善, 温庆忠. 滇西北玉龙雪山不同海拔云南松 (Pinus yunnanensis) 径向生长对气候因子的响应. 生态学报, 2018, 38(24) : 8983-8991.

[ 2 ] 燕兴国. 玉龙雪山白水河 1 号冰川物质平衡与表面运动速度研究 [D ]. 兰州: 西北师范大学, 2018.

[ 3 ] 何慧娟, 王钊, 董金芳, 王娟. 基于 MODIS 产品的秦岭地区 NDVI、地表温度和蒸散变化关系分析. 西北林学院学报, 2019, 34(4)：179184, 191- 191 . 
[ 4 ] 黄秀华, 李正文, 关燕宁, 荆林海. 热红外遥感找油的时效性研究. 遥感学报, 2003, 7(2): 142-145.

[ 5 ] 张小飞, 王仰麟, 吴健生, 李卫锋, 李正国. 城市地域地表温度-植被覆盖定量关系分析一一以深圳市为例. 地理研究, 2006, 25(3)： 369-377, 561-561.

[ 6 ] 毛克彪, 唐华俊, 周清波, 陈仲新, 陈佑启, 覃志豪. 用辐射传输方程从 MODIS 数据中反演地表温度的方法. 兰州大学学报: 自然科学 版, 2007, 43(4): 12-17.

[7] 周纪, 陈云浩, 李京, 翁齐浩, 易文斌. 基于遥感影像的城市热岛容量模型及其应用一一以北京地区为例. 遥感学报, 2008, 12(5)： 734- 742 .

[ 8 ] 徐永明, 覃志豪, 沈艳. 基于 MODIS 数据的长江三角洲地区近地表气温遥感反演. 农业工程学报, 2011, 27(9): 63-68.

[ 9 ] Brabyn L, Zawar-Reza P, Stichbury G, Cary C, Storey B, Laughlin D C, Katurji M. Accuracy assessment of land surface temperature retrievals from Landsat 7 ETM+in the Dry Valleys of Antarctica using iButton temperature loggers and weather station data. Environmental Monitoring and Assessment, 2014, 186(4) : 2619-2628.

[10］卢显, 孟庆岩, 顾行发, 张晓东, 马未宇. 基于 HJ-1B 卫星的玉树 $M_{\mathrm{S}} 7.1$ 地震地表温度异常识别. 中国地震, 2015, 31(4) : 679-687.

[11] 冯海英, 冯仲科. 基于 MODIS LST 产品的山东省森林调节温度生态服务价值评估新方法. 林业科学, 2018, 54(2) : 10-17.

[12] 吴文渊, 金城, 庞毓雯, 赵丽佳, 宋瑜, 胡潭高, 张登荣, 徐俊锋. 热红外遥感浙江地表热环境分布研究. 遥感学报, 2019, 23 (4) : 796-807.

[13] 张祖勋, 张剑清. 数字摄影测量学. 武汉: 武汉测绘科技大学出版社, 1996 .

[14] 赵敏, 林行刚, 赵乃国, 王倩.一种 DSM 模型下飞行障碍物尺度的研究. 清华大学学报：自然科学版, 2003, 43(9)：1249-1252.

[15] 王明华. 工程岩体三维地质建模与可视化研究 [D]. 武汉: 中国科学院研究生院(武汉岩土力学研究所), 2004.

[16] Friman O, Tolt G, Ahlberg J. Illumination and shadow compensation of hyperspectral images using a digital surface model and non-linear least squares estimation//Proceedings of SPIE 8180, Image and Signal Processing for Remote Sensing XVII. Prague, Czech Republic: SPIE, 2011 : 19.

[17] Brédif M, Tournaire O, Vallet B, Champion N. Extracting polygonal building footprints from digital surface models: a fully-automatic global optimization framework. ISPRS Journal of Photogrammetry and Remote Sensing, 2013, 77: 57-65.

[18] Sander L, Raniolo L A, Alberdi E, Pejrup M. Elevation trends in wide beach-ridge systems retrieved from Landsat Images and the SRTM Digital surface model. Journal of Coastal Research, 2015, 31(5): 1241-1252.

[19] 凌成星, 鞠洪波, 刘华, 张怀清, 孙华.一种基于 GeoEye- 1 立体像对数据的快速提取平均树高方法. 测绘与空间地理信息, 2018 , 41 (3) : 7-10

[20] 解金卫, 索志勇, 李真芳, 王跃锟. 基于 PolInSAR 的植被区高精度数字表面模型反演方法. 电子与信息学报, 2019, 41(2): 293-301.

[21] Jiménez-Muñoz J C, Sobrino J A. A generalized single-channel method for retrieving land surface temperature from remote sensing data. Journal of Geophysical Research, 2003, 108(D22): 4688.

[22] 蒋大林, 匡鸿海, 曹晓峰, 黄艺, 李发荣. 基于 Landsat 8 的地表温度反演算法研究一一以滇池流域为例. 遥感技术与应用, 2015, 30(3)： 448-454.

[23] Zhang Y F, Zhang Y J, Mo D L, Zhang Y, Li X. Direct digital surface model generation by semi-global vertical line locus matching. Remote Sensing, 2017, 9(3): 214 .

[24] 冀琴, 董军, 刘睿, 肖作林, 杨太保. 1990-2015 年喜马拉雅山冰川变化的遥感监测及动因分析. 地理科学, 2020, 40(3): 486-496. 